\title{
Ethical issues raised by the introduction of payment for performance in France
}

\author{
Olivier Saint-Lary, ${ }^{1,3}$ Isabelle Plu, ${ }^{2}$ Michel Naiditch ${ }^{3}$
}

${ }^{1}$ Medecine Generale, UVSO,

Guyancourt, France

${ }^{2}$ Médecine Légale, Paris

Descartes, Paris, France

${ }^{3}$ Prospere, IRDES, Paris, France

Correspondence to Dr Olivier Saint-Lary, Medecine Generale, UVSQ, 9 bd d'alembert, Guyancourt 78280, France;

oliviersaintlary@hotmail.com

Received 3 August 2011 Revised 16 February 2012 Accepted 23 February 2012

\begin{abstract}
Context In France, a new payment for performance (P4P) scheme for primary care physicians was introduced in 2009 through the 'Contract for Improving Individual Practice' programme. Its objective was to reduce healthcare expenditures while enhancing improvement in guidelines' observance. Nevertheless, in all countries where the scheme was implemented, it raised several concerns in the domain of professional ethics.
\end{abstract}

Objective To draw out in France the ethical tensions arising in the general practitioner's (GP) profession linked to the introduction of P4P.

Method Qualitative research using two focus groups: first one with a sample of GPs who joined P4P and second one with those who did not. All collective interviews were recorded and fully transcribed. An inductive analysis of thematic content with construction of categories was conducted. All the data were triangulated.

Results All participants agreed that conflicts of interest were a real issue, leading to the resurgence of doctor's dirigisme, which could be detrimental for patient's autonomy. GPs who did not join P4P believed that the scheme would lead to patient's selection while those who joined P4P did not. The level of the maximal bonus of the P4P was considered low by all GPs. This was considered as an offense by non-participating GPs, whereas for participating ones, this low level minimised the risk of patient's selection.

Conclusion This work identified several areas of ethical tension, some being different from those previously described in other countries. The authors discuss the potential impact of institutional contexts and variability of implementation processes on shaping these differences.

\section{INTRODUCTION}

Primary care physician Pay for Performance (P4P) programmes have developed widely over the last 10 years. Countries such as the USA, Canada, Australia, New Zealand, Israel and The Netherlands have tested them through various experimental designs. Yet, the only P4P programme set up at national level is the 'Quality and Outcomes Framework' (QOF) implemented in the UK in 2004. In France, a similar programme called 'Contract for Improving Individual Practice' (CAPI) ${ }^{2}$ was introduced, on a voluntary basis, in 2009 by the Caisse Nationale d'Assurance Maladie pour les Travailleurs Salariés (CNAMTS), the National Health Insurance Fund (NHIF) for salaried workers.
The CNAMTS's main objective was, just as that of the QOF, to improve the quality of care. But the context in which each scheme was introduced was highly contrasted: the National Health Service (NHS) agreed to pay the price of practice bonuses linked to the new P4P scheme, which represents only an element of the overall cost of the new GMS contract for general practitioners (GPs). The overall cost was $£ 8$ billion in the first 3 years. ${ }^{3}$ The NHS counts upon other instruments for cost containment. ${ }^{4}$ In contrast, although the French NHIF that designed the CAPI programme is supposed to deal with all ambulatory health costs, the way the CAPI scheme was designed shows that the 'quality objective' was expected to be reached in (at least) a cost neutral way. Indeed, the NHIF expected a zero implementation cost (which it almost achieved) on the premise that savings, arising mostly from an increase in 'generic prescriptions', would cover the bonus $\operatorname{costs}^{5}$ while improvement in preventive care through better screening and greater use of guidelines would entail a better overall performance. But an indirect expected cost saving effect was that these bonuses contributed to stopping French physicians' pressure on service prices. Indeed, contrary to GPs in the UK, who are mostly paid through capitation, the largest part of French GPs' revenues comes from fees-for-services (FFS), which raises some ethical issues such as 'induced demand'. 67

The CAPI programme is based on a group of 16 practice indicators (box 1). A GP's bonus payment results from a formula mixing the level of achievement and of improvements in practice performance from 1 year to the next. The maximum amount that can be earned in a year is approximately $€ 5000$ (about $90 \%$ of a GP's monthly average net revenue). Six months after its introduction, almost 12600 contracts had already been signed at national level, that is, around a third of eligible physicians joined the programme. ${ }^{5}$

This figure may be considered as a success given that the CAPI system, when it was announced, was met by almost universal and fierce opposition mostly from the doctors' unions. They criticised the scheme for it had been set up without consulting the medical profession representative organisations or academics. This lack of consultation was also felt as a potential threat to the general agreement negotiating process which sets common goals between the physicians' regulating body (NHIF) and unions. 'Opinion leaders' (through their blogs) and the 'National Council of 


\section{Box 1 The 16 current Contract for Improving Individual Practice programme indicators}

1. Influenza. Patients aged over 65 vaccinated/Patients over 65.

2. Breast cancer. Women from 50 to 74 years old participating in breast cancer screening/Women from 50 to 74 years of age (calculated on 2 years).

3. Vasodilators. Patients over 65 treated/Patients over 65 (Target=decrease).

4. Benzodiazepines long half-life. Patients over 65 treated/Patients over 65 (Target=decrease).

\section{Diabetes}

5. Number of diabetic patients with 3 or $4 \mathrm{HbA} 1 \mathrm{C}$ per year/number of diabetic patients.

6. Number of diabetic patients with ophthalmological control in the year/number of diabetic patients.

7. Number of diabetic patients (men over 50, women over 60) treated with statins and antihypertensive/number of diabetic patients (men over 50, women over 60) treated with antihypertensive drugs.

8. Number of diabetic patients (men over 50, women over 60) treated with antihypertensive drugs, statins and aspirin low dose/number of diabetic patients (men over 50, women over 60) treated with antihypertensive and statins.

9. Patients treated with antihypertensive normalised their blood pressure below 140/90 (declarative).

\section{Prescription}

10. Per cent of generics for antibiotics.

11. Per cent of generics for proton pump inhibitor.

12. Per cent of generics for statins.

13. Per cent of generics for antihypertensive drugs.

14. Per cent of generics for antidepressants.

15. Prescription of ACE inhibitors/prescription of ACE inhibitors + angiotensin II receptor antagonists.

16. Number of patients treated with low-dose aspirin/number of patients treated with antiplatelet agents

the Order of Physicians', which is responsible for the profession guiding principles of clinical practice, also expressed their opposition to the scheme. Among other arguments, both stakeholders contended that the CAPI principles and logic were contrary to the ethics of the medical profession and that the physicians who had signed the contract would face possible conflicts of interest.

The emergence of ethical issues did not come as a surprise as several Anglo-Saxon review articles and opinion pieces had already evoked a number of ethical tensions inherent to $\mathrm{P}_{4} \mathrm{P}^{8-11}$ such as: information bias, missed reporting, adverse selection leading to inequity and neglect of unmeasured areas in $\mathrm{P} 4 \mathrm{P}$ programmes, but also erosion of doctors' intrinsic motivation. These tensions call to mind Amartya Sen's opinion when he states that 'the economic legitimacy of activities whose ultimate aim is to improve public health inevitably raises the question of ethics, which must be 're-embedded into economics as a moral science, 12

The main objective of this study is to identify the ethical tensions that arose in the GP profession in France as an outcome of the introduction of the CAPI ambulatory care P4P programme and to compare them with the ones already spotted in the UK's QOF.

The rationale for this goal comes from the fact that, despite a common professional culture and training, the specific institutional context in which French doctors are working and differences in the design and implementation processes of the CAPI programme are likely to change the relative weight of these ethical concerns and/or give rise to new ones.

The CAPI design using a 'generic prescription indicator' was not considered useful for the QOF as UK's GPs had long been encouraged to prescribe generics and had reached a good rate of prescription (much higher than that of French GPs). ${ }^{13}$ Also, unlike the QOF design, the CAPI programme precludes the exclusion of certain patients for specific indicators when calculating performance levels.
Regarding the implementation of the CAPI programme, the choice of indicators and their levels as well as the formula for bonuses was unilaterally fixed by the NHIF without formally consulting GPs' scientific groups or unions. French patients were not 'officially' informed by the NHIF of the introduction of P4P. There was also no obligation for GPs to inform their patients about whether they were or not participating in the CAPI programme. Consequently, a patient, if not informed by his/her GP, could not be aware of whether his/her attending physician had signed a CAPI contract but also of his/her doctor's level of performance as there were no public disclosures of the results.

Regarding influential institutional factors, the already-stated payment methods, $46 \%$ of French GPs are in solo-practice. ${ }^{14}$ And unlike British patients who have to register with a practice and tend to stay with their GP for a long time, French patients are still allowed to change GPs without any financial penalty (although 92\% in 2010 picked their 'primary doctor' (médecin traitant) in order to improve their healthcare pathway and to be better reimbursed). ${ }^{15}$

\section{METHOD}

A qualitative study led by focus groups seemed more appropriate to highlight ethical tensions. According to Morgan, ideas are constructed through the social process of communication. ${ }^{16}$ The focus group places participants in a social interactive context that stimulates and encourages the free expression of ideas and opinions by the ratchet effect.

\section{Focus group recruitment}

Two focus groups were organised; the first with only GPs who had signed a CAPI contract (named CAPI+) and the second with only non-participating GPs (CAPI-). The objective was to gather two samples of GPs reasoned for gender, age, and length of time in general practice and practice location. Recruitment followed a two-step process: the first step was to contact GPs in 
the Ile de France region through random telephone calls. The second step, if necessary, was to try to reach them using two lists of email addresses.

The group of CAPI- was selected through the first step.

But, as it turned out, the number of CAPI volunteers remained too small, in spite of our recourse to the second step, and it was necessary to solicit new contacts. They were recruited for convenience by using a 'snow ball' technique. We thus expected the CAPI+ group to be biased with respect to our selected criteria.

Besides, participants were rewarded with a small payment $(€ 100)$.

\section{Focus group management}

The focus group interviews were moderated by a sociologist following structured guidelines set up by a multidisciplinary team of four researchers (a public health physician, a research professor in medical ethics, a GP and the sociologist himself). The guidelines were elaborated in a way that avoided directly suggesting potential ethical dilemmas and enabled them to emerge spontaneously during the interviews. However, if a specific issue within the focus guidelines (see appendix 1) was not spontaneously broached during the interviews, it was put up to the group in order to gauge how the participants reacted to it. There was no time limit to the meeting, which ended when the researchers estimated that the debate had reached its 'saturation point'.

\section{Data analysis}

Group interviews were recorded with the permission of the participants and integrally transcribed. An inductive thematic analysis was then conducted with the themes that emerged from a content analysis of the participants' declarations. The data transcripts were read several times with a choice of units of meaning, the identification of themes, categorisation and classification. The data were then validated through cross verification from the four researchers to optimise data conformity.

\section{RESULTS \\ Participant profiles}

In the CAPI+ group, the participants' profiles were relatively homogeneous: six (two did not attend) male subjects, between 38 and 52 years old, in urban areas. The majority (4/6) were intern supervisors. The CAPI- group (eight) was more heterogeneous. It was composed of three women and five men from 32 to 60 years of age. One GP practiced in a semirural zone and the others in urban areas. Only one also had teaching activities.

\section{Discussion content}

The analysis revealed that the influence of the new scheme on medical practices raised ethical issues along two main dimensions, the doctor-patient relationship and GP's relation to money.

\section{The doctor-patient relationship}

The CAPI+ physicians used a paradoxical argument to justify their opinion that the scheme had little impact on their practice: they pretended to have only a weak knowledge of the indicator so that the introduction of the new scheme could account for no real change, adding that they indeed had experienced none. However, after a short debate, several CAPI+ GPs acknowledged that the CAPI programme could have an impact on their practices by comparing its potential impact with that induced by visits of pharmaceutical company representatives.

We like to think that as private practitioners, it has no effect on us, we like to think that it doesn't have any influence on us, in the same way that medical representatives and advertising have no influence on us. A GP is supposedly impervious to all that. I still think that when we receive our indicators it leaves an impact, and even if we're not necessarily conscious of the fact, it nonetheless leaves an impact somewhere' (CAPI+, GP 2).

With regard to changes in the patient-doctor relationship, three specific issues arose: loss of trust, lack of respect for the patient's autonomy and a potential conflict of interest.

GPs in the CAPI- group predominantly believed that the trust which lies at the heart of the patient/doctor relationship could be undermined by the CAPI logic.

'A patient goes to see a doctor partly because he needs to see a doctor and partly because he trusts the doctor he goes to see. If the GP has this invisible or virtual third party sitting beside him saying 'no, no you're to remove that drug from the prescription...' it's no longer a relationship based on trust' (CAPI-, GP 4).

The risk of 'trust loss' was not perceived in the same way by both groups as shown by the following example: CAPI- GPs felt uncomfortable that patients were not informed as to whether their GP had signed the contract and felt this would contribute to a deterioration of trust. Conversely, CAPI + GPs did not share this view, first because they considered CAPI indicators as scientifically validated guiding principles that they were following even before the P4P scheme was implemented, but also because of their views regarding information and patient autonomy (see below).

This difference was in line with the specific views of each group on patient information and autonomy. CAPI- doctors felt that patient autonomy could be at risk, for example, in cases of a patient's non-compliance with the goals set in a CAPI indicator, his/her GP, in order to avoid a loss of income, could be inclined to more interventionism to counter the patient's preferences:

'But now that they've brought me the figures and that I've reached $30-40 \%$ of the target, I said to myself that I would be careful and if in addition I lecture them (patients) a bit, maybe it will be to my benefit' (CAPI-, GP 5).

This was not the case for CAPI+ doctors who consider that informing is by essence never neutral as information must contribute to patient compliance:

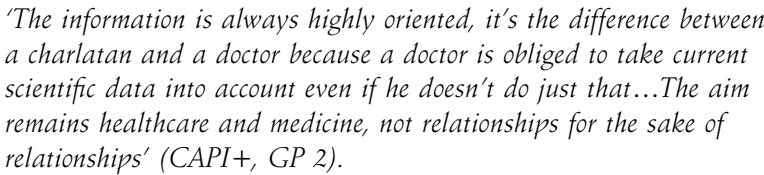

Thus, the clinical information they provided to their patients did not change; they considered that not informing their patients about their CAPI status could not be seen as a threat for the patient-doctor trust.

GPs in both groups agreed that the financial incentives linked to the CAPI logic could lead to a conflict of interests undermining the patient/doctor relationship:

'You don't have the same objectives when you want to provide health care, real health care, you don't have the same objectives when you want to deal with the economics of health' (CAPI-, GP 2).

But the two groups had opposing views regarding its weight. This was clear when GPs mentioned 'risk selection', which they 
considered as the maximum negative outcome of this conflict. CAPI- GPs clearly quoted the danger of excluding the least compliant patients, while none of the CAPI+ GPs shared this concern as they thought that the FFS system prevented this risk:

'So far, I have had the impression that it was the patients who chose us and not the opposite' (CAPI+, GP 2).

Nevertheless, they agreed that this selection risk could eventually exist but only for a much higher amount of money.

\section{Relationship to money}

The main goal was to examine how the bonus linked to the CAPI scheme fitted into the previous FFS scheme and to which ethical problems it could lead.

For the majority of GPs in both groups, the dominant FFS system of payment in France was judged unsatisfactory as it induced GPs to spend less time with complicated patients and was judged as being unfair

\section{'But fee-for-service payment does not fit with our practice: it is not the same amount of work and thus time that you would need when you care for an addict and/or an hepatitis $C$ patient compared to a patient with an upper respiratory tract infection. But the payment is the same: is it fair? (CAPI+, GP2).}

Being paid solely according to consultation number was considered as inappropriate by both groups but never explicitly identified as a threat to good practice. This apparent contradiction may be linked to the fact that, in both groups, doctors were not inclined to abandon FFS, which they considered a guarantee for their revenue. While this judgement logically entails that they can increase revenue by enhancing their activity, none of them explicitly mentioned that this increase could be a threat to quality (eg, by providing unnecessary care).

Nevertheless, in both groups, almost all the participants contended the CAPI logic-increasing GPs' income in order to improve their practice-could be perceived by the general public as publicly signalling a 'breach of the professional code of ethics'.

'What does that mean; it means that to be a good medical practitioner one has to pay you even more' (CAPI+, GP 3).

We also noticed differences in opinion regarding the bonus level: non-signatory GPs judged the reward as insulting because it was so small that it could not account for the value of GPs' work. While all CAPI+ GPs, although they also considered the bonus as being low, declared nevertheless that it represented a first recognition by the CNAM that their practice was adequate:

'For once we're being rewarded for good medical practice, at least what I consider to be good medical practice, because we're going to spend 5 minutes more, because we're going to deal with a host of clinical examinations because the recommendations require it' (CAPI+, GP 3).

Doctors in both groups judged that the low level of the bonus (when compared with their revenue) excluded the risk of practice abuse

'So that I am going to be slightly provocative: up to 50000 Euros I have moral values! Beyond that um... (Laughter)' (CAPI-, GP 4).

More interestingly, the fact that the bonus level is dependent on target attainment was perceived by the CAPI- group as a possible source of work-related stress.

'In relation to targets in general, patients who suffer in their work environment do so because of target agreements fixed by companies each year and that effectively change every year to overlap and accumulate somewhat. This causes a great deal of suffering' (CAPI-, GP 2).

In contrast, because they considered themselves to be in line with 'CAPI targets', CAPI+ doctors did not feel pressed and thus at risk of stress by the CAPI indicator levels.

\section{DISCUSSION \\ The CAPI contract, patients' right to be informed and relationship to care}

From a legal standpoint, there is no obligation for GPs to inform their patients that they are involved in the CAPI programme (just as there is no requirement for GPs to inform their patients of how many pharmaceutical representatives they have seen in the past month). The risk that P4P may favour a drift towards a more 'paternalistic doctor-patient relationship' has already been identified in the medical literature. ${ }^{10} 11$

From these group interviews it appeared, and this seems logical, that most CAPI+ physicians have few qualms about applying recommendations driven by financial incentives without informing their patients about their CAPI status, as they assume these recommendations are beneficial for their patients.

The second reason why CAPI+ GPs think that giving patients information about the CAPI status is not useful is that they consider that any health information delivered during a medical consultation is, by essence, never neutral and always biased. Indeed, the main objective of this information is to reinforce patients' compliance, which can lead to reducing patients capability to do what the GPs do not want them to do. According to most CAPI+ GPs, patient information is not a guarantee of patient autonomy. Even worse, for some of these GPs it may give patients a false feeling of autonomy, which they consider as hypocrisy.

This indicates their clear tendency towards medical paternalism, which paradoxically disregards the content of the Law of 4 March $2002^{17}$ that places patients at the core of any medical decision by promulgating the principle of consent and autonomy based on the right to be fully informed. In this regard, the way the CAPI system has been elaborated and implemented (which also clearly goes against these principles) is troubling both groups.

If patient information concerning health status, the disclosure of a disease, the prescription of a new medical treatment or the need for a surgical procedure is essential, things are not so clear as regards information about the CAPI status of a physician.

Two questions arise here: Why inform? And how to inform?

\section{Why inform?}

There are classically four ethical principles which impact upon the concept of consent to medical treatment: the patient's right to independent thought and decision making (principle of autonomy), the doctor's duty to act in the best interests of the patient (principle of beneficence), the oath of Hippocrates primum non nocere (principle of non-maleficence) and the questions of distribution of healthcare resources (principle of justice). ${ }^{18}$

The CAPI programme promotes both principles of beneficence and non-maleficence by encouraging physicians to respect guidelines, which, as they are supposed to be based on the best scientific evidence, should be beneficial to patients. In a certain way, it also fosters the justice principle by limiting health expenditures (but only if the surpluses are used in a fair way). 
But the CAPI programme may question the autonomy principle by encouraging the physician to focus on the indicators' objectives at the cost of limiting or denying patients' expectations. This raises the following question: should the autonomy principle dictate the information process and content?

Many philosophical ethical theories have sought to justify the importance of the principle of autonomy. Probably the most influential one is Kant's theory ${ }^{19}$ which indicates that rational human beings could identify moral duties according to what he calls a 'categorical imperative'. The essence of Kant's categorical imperative is that people possess intrinsic moral worth and are entitled to equal consideration. The respect for autonomy is therefore, at least, an acknowledgement of a person's right to make his/her own life decisions and hold his/her own views based on personal values and beliefs without interference.

According to this theory, the patient whose health is at issue should be completely informed on factors likely to influence his/ her physician's prescriptions.

Nevertheless, the autonomy of patients who have chosen, in all awareness, a CAPI+ regular GP may be endangered: these patients could think they are trapped by this choice that 'logically' forces them to consent to prescriptions or medical examinations included in the CAPI indicators, which, indirectly, would undermine their autonomy. The paradoxical consequence of this point of view would be that an 'uninformed patient' would have a larger freedom to oppose his/her GP's prescription. However, this paradox perhaps appeared because CAPI+ GPs seemed to be more directive than CAPI- GPs in our study. Even if they did not elaborate as far as that, it seems that CAPI+ GPs considered the beneficence principle as the most important one, thus undermining the others and leading to incomplete or biased information.

We can suppose that if GPs' characteristics regarding information were the same in both groups, patient autonomy would not be undermined because of an informed choice to see a GP who operates under the CAPI system. An informed patient could therefore discuss with his/her GP about the opportunity to prescribe such or such drug or such or such blood test. If information is complete and neutral, and gives patients the choice to do or not what the physician advises, patient autonomy will be preserved.

\section{How to inform?}

From our point of view, patients' oral specific and systematic information during the time of the medical consultation does not seem suitable. The medical consultation time should be dedicated to listening to patients' troubles and not designed for physicians to speak about their practice. The information has to be comprehensive; the main CAPI objective, which is the improvement of the quality of care, has to be pointed out, for instance, with information posted in the waiting room. Information leaflets could be left for patients' use in GPs' waiting rooms, with for instance a list of internet sites for patients who want to learn more about it.

\section{Emergence of new conflicts of interest?}

A conflict of interests arises from a situation in which a GP's personal interests are at odds with the mission of delivering optimal care he/she is entrusted with. Even without proof of prejudicial acts, such a situation could create a doubt as to a GP's capacity to exercise his/her mission with complete objectivity. In our study, both groups recognised that the CAPI scheme could convey potential conflicts of interests but the perception of opportunities for such conflicts to emerge differed in the two groups.
Informing patients about the CAPI status of GPs does not prevent conflicts of interests, as it allows patients to take this situation into account before deciding for their own health. Not informing them does not increase the risk of conflicts of interests, but puts the GPs under the suspicion of being attracted, not by good medical practice, but by the lure of profit.

The debate in the UK regarding the measles, mumps and rubella vaccine illustrates this case: a non-evidenced and thus unfounded belief linked vaccine injections with the potential development of autism. The fact that UK doctors received bonuses based on their vaccination coverage rates led many patients to believe that GPs were deliberately withholding information on the vaccine impact in order to increase their vaccination rates and thus their revenue. This led to such a loss of trust in GPs' professional behaviour that UK GPs finally requested that the vaccination indicator for children be removed. ${ }^{9}$

The acuity of this conflict was not equally perceived by the two groups: the CAPI- group saw the National Health Insurance's economic objectives as radically opposed to GPs' quality of care objectives. The CAPI+ GPs believed that, even if each stakeholder's objectives were different, they were not necessarily incompatible as a GP is also an economic agent who cannot ignore the economic constraints the health system is faced with. They admitted that because GPs have a 'natural tendency' to overprescribe, it is 'natural' for the regulator to introduce rules to control public expenditures. Choosing to participate in the CAPI scheme, the logic of which entails economic constraints (such as prescribing less costly generic drugs), does not necessarily lead to conflict with the values of GPs as long as the indicators and the targets are well designed. That is why several GPs in the CAPI+ group judged (contrary to all GP- doctors) that conflicts of interests did not arise directly from the pay for performance principle but rather from the nature of the clinical indicators selected and the fact that objectives were unilaterally determined by the Health Insurance Fund. Consequently, despite their opposed opinions regarding the P4P principle, the two groups reached a consensus on the need to involve GPs in setting up clinical indicators to avoid too large gaps between healthcare values and the values that govern healthcare cost containment.

\section{Towards a risk of patients' selection?}

The CAPI- group feared that the most likely victims of patients' selection would be the most precarious populations. A tool aimed to improve the quality of public health, including equity and fairness, could thus paradoxically increase health inequalities by excluding the most vulnerable populations. The CAPI+ GPs did not spontaneously evoke the danger of patients' selection, although it is described in the literature. ${ }^{20}{ }^{21}$ Nevertheless, they recognised that it could occur if the bonus associated with target achievements was to increase. In future, the possible exclusion of certain categories of patients from CAPI calculations of practice achievement while retaining them on signatory GPs' patient registers could be considered. This has been introduced in the form of 'exception-reporting' in the QOF in the UK. The average rate of exclusion remains below $6 \%$ and represents only $1.5 \%$ of the cost of the programme. ${ }^{22}$ In addition, it appears that the QOF programme has succeeded in reducing health inequalities, ${ }^{23}$ at least on certain indicators.

\section{Is there a risk of GPs concentrating exclusively on the measured indicators?}

This risk of neglecting clinical areas that are not incentivised, although discussed in the $\mathrm{UK}^{24}{ }^{25}$ was not evoked 
Box 2 Main differences in ethical issues regarding payment for performance (P4P) between France and England based on French general practitioners' (GPs) opinion

- Patients are not aware of P4P existence and more specifically have no information regarding the participation of their GP and their level of performance.

- The lack of exception-reporting might increase the risk of patient selection.

- A higher risk of conflict of interest due to the predominant role of the Caisse Nationale d'Assurance Maladie pour les Travailleurs Salariés (CNAMTS) in the design and implementation of the system. Particularly because of the two objectives of CNAMTS: improving quality of care and cost containment.

- No possibility of misreporting as the CNAMTS controls all the indicator data.

- Few chances to neglect unmeasured areas of clinical practice because of the very limited scope of the Contract for Improving Individual Practice programme's indicators.

- Low P4P bonus might minimise the risk of unethical practice for financial motivations.

spontaneously in the focus groups. The explanation is that contrary to the QOF system, the range of medical activities targeted by the CAPI indicators is relatively narrow. It thus appeared impossible for the GPs to distance themselves from the other areas of medical care, given the narrow field covered by CAPI indicators.

\section{Study limits and perspectives}

The study's main limits concern the existence of a selection bias in the CAPI+ participants due to the recruitment process and the limited number of GPs in each group which results from that process. There is therefore a risk that some perspectives on both sides of this debate were not captured and that there might be more diversity in physicians' judgement related to ethical tensions.

The aim of separating the two groups was to avoid the possibility of a 'sterile' debate in the case of profound disagreement between signatories and non-signatories, which could be expected from the strong opposition between supporters of and opponents to the scheme. Therefore, neither a random nor a purposive sampling technique could be used for recruitment. The limited number of the CAPI- group did not, in our opinion, lead to major bias. The reasons for this are: GPs were easily recruited, showed great heterogeneity of opinion with initial divergent opinions on many issues and did not reach consensus at the end of the discussion for some of them. The limited number of the CAPI+ group is linked to the following facts: according to the chosen process, as only two CAPI+ GPs had accepted to participate after we processed more than 100 telephone calls and sent 2000 emails from a list of the French Society of General Practice in Ile de France, we used the snow ball technique, which allowed us to recruit six more participants. Among them, two did not show up the day of the focus group, thus leaving us with six CAPI+ GPs. Thus, if consensus in this group was more frequent for major issues, we cannot reject the hypothesis that this procedure conveyed a risk of opinion bias.

Two complementary hypotheses can be put forward to explain difficulties in recruiting CAPI+ doctors that may help interpreting our findings. First, it is easier to mobilise individuals when they are opposed to something, whatever the issue. Second, and more important, many GPs (among whom CAPIGPs) are still extremely suspicious of the NHIF that promoted and designed the CAPI programme. So, their negative opinion of the CAPI design may be linked to its perceived logic and to the imputed intention of its designer, as the doctor who agrees to participate in CAPI may be looked at by the majority of the profession as an NHI 'ally', currently a difficult position for a French GP to assume.

\section{CONCLUSION}

Driven by public health and economic concerns, the French health authorities have introduced a new P4P scheme which clearly has modified the context in which the doctor-patient relationship is embedded. Our results show that GPs believe that modifying the ways they are paid has led to a modification in the way patients perceive them. For GPs who refused this scheme, P4P raised a number of issues linked to patient autonomy while placing the GP at the centre of conflicting interests. In connection with this conflict, they identified several areas of ethical tension, some different from those previously described in the literature, because of differences in professional context and the implementation process (box 2).

Most signatories GPs considered P4P as rewarding good practice and complementary of FFS. They did not necessarily consider that these schemes led to conflict of interests as long as the indicators were well designed and the financial bonus considered as low.

However, for a higher amount, both groups agreed that conflicts of interests may occur.

They might be inherent to the principle of P4P but GPs also mentioned that these tensions might be enhanced by the way CAPI indicators have been constructed in France. It remains to see if involving GPs in their design, as proposed by some physicians, is likely to ensure that target achievements will guarantee patients' rights regarding their autonomy without renouncing the values and the professional code of ethics that govern medical practice.

Acknowledgements Philippe Boisnault, Philippe Szidon, Didier Duhot, Pascal Clerc, Thomas Cartier, Nathalie Pelletier Fleury, Carine Franc, Nicolas Krucien, Marc Le Vaillant, Julien Mousques, Cecile Fournier, Philippe Le Fur, Yann Bourgeuil (Prospere team). We also acknowledge Larry Brown Christian Hervé and lona Heath.

Contributors OSL: conception, design, analysis and interpretation of data; IP: conception, design, revising it critically for important intellectual content; MN: analysis and interpretation of data, final approval of the version to be published.

\section{Competing interests None.}

Provenance and peer review Not commissioned; externally peer reviewed.

\section{REFERENCES}

1. Doran T, Fullwood C, Gravelle $\mathrm{H}$, et al. Pay-for-performance programs in family practices in the United Kingdom. N Engl J Med 2006;355:375-84. 
2. Décision du 9 mars 2009 de I'Union nationale des caisses d'assurance maladie relative à la création d'un contrat type d'amélioration des pratiques à destination des médecins libéraux conventionnés. [Decision of 9 March 2009 from the National Union of sickness funds relating to the creation of a standard contract of improvement practices for liberals' physicians under agreement]. JORF n0093 du 21 avril 2009 page 6839, texte $n^{\circ} 34$.

3. Walker S, Mason AR, Claxton K, et al. Value for money and the Quality and Outcomes Framework in primary care in the UK NHS. Br J Gen Pract 2010;60:213-20.

4. Walley T, Mrazek M, Mossialos E. Regulating pharmaceutical markets: improving efficiency and controlling costs in the UK. Int J Health Plann Manage 2005:20:375-98.

5. Anon. Les CAPI ont séduit $30 \%$ de leur cible, 12566 contrats signés en six mois. Le quotidien du médecin 2009;8676:3.

6. Fuchs VR. Physician-induced demand: a parable. J Health Econ 1986:5:367.

7. Rochaix L, Jacobzone S. L'hypothèse de demande induite: un bilan économique. Economie et Prévision 1997;129-130:25-36.

8. Heath I, Hippisley-Cox J, Smeeth L. Measuring performance and missing the point? BMJ 2007;335:1075-6.

9. Slowther A, Ford S, Schofield T. Ethics of evidence based medicine in the primary care setting. J Med Ethics 2004;30:151-5.

10. Snyder L, Neubauer RL. Pay-for-performance principles that promote patientcentered care: an ethics manifesto. Ann Intern Med 2007;147:792-4.

11. Mannion R, Davies HTO. Payment for performance in health care. BMJ 2008;336:306-8.

12. Sen A. Léconomie est une science morale. Paris: La Découverte/Poche, 2003.

13. Duerden MG, Hughes DA. Generic and therapeutic substitutions in the UK: are they a good thing? Br J Clin Pharmacol 2010;70:335-41.

14. Baudier $\mathbf{F}$, Bourgueil $Y$, Evard I, et al. Group practice dynamics among private general practitioners from 1998 to 2009. Questions d'économie de la santé Irdes 2010;157. http://www.irdes.fr/EspaceAnglais/Publications/IrdesPublications/ QES157.pdf

15. Dourgnon P, Naiditch M. The preferred doctor scheme: a political reading of a French experiment of gate-keeping. Health Policy 2010;94:129-34.

16. Morgan DL. Focus Group as Qualitative Research. Londres: Sage, 1997.

17. Loi du 4 mars. 2002. http://www.legifrance.gouv.fr/affichTexte.do? cidTexte $=$ JORFTEXT000000227015 (cité 14 avr 2011)

18. Beauchamp T, Childress J. Principles of Biomedical Ethics. 6th edn. New York: Oxford University Press, 2009.

19. Heubel F, Biller-Andorno N. The contribution of Kantian moral theory to contemporary medical ethics: a critical analysis. Med Health Care Philos 2005; 8:5-18.

20. Karve AM, Ou F, Lytle BL, et al. Potential unintended financial consequences of pay-for-performance on the quality of care for minority patients. Am Heart $J$ 2008; 155:571-6.

21. Shen Y. Selection incentives in a performance-based contracting system. Health Serv Res 2003;38:535-52.

22. Campbell S, Hannon K, Lester H. Exception reporting in the Quality and Outcomes Framework: views of practice staff-a qualitative study. $\mathrm{Br} \mathrm{J}$ Gen Pract 2011;61:183-9

23. Doran T, Fullwood C, Kontopantelis E, et al. Effect of financial incentives on inequalities in the delivery of primary clinical care in England: analysis of clinica activity indicators for the quality and outcomes framework. Lancet 2008;372:728-36

24. Campbell SM, McDonald R, Lester H. The experience of pay for performance in English family practice: a qualitative study. Ann Fam Med 2008;6:228-34.

25. Heath I, Rubinstein A, Stange KC, et al. Quality in primary health care: a multidimensional approach to complexity. BMJ 2009;338:b1242.

\section{APPENDIX 1 \\ Focus Groups' interview guides} Interview guide for CAPI + GPs

Presentation: This research aims (1) to analyze your perception and knowledge as doctors who joined the scheme soon after it was launched. (2) To analyze if and how the scheme had an impact on your clinical practice.

First each participant will be invited to present himself. I suggest you tell us:

1. Your practice location

2. Your practice setting (solo, group practice)

3. The profile of your patients

4. Since when you have been practicing

5. What led you to choose general practice?

6. Other activities linked to doctoring (unions, teaching...)

What were your main motivations to join the CAPI scheme?

How did you perceive it?

What do you think the objectives of the scheme are?

After an overall discussion to see their general perception of the scheme we proceed to a more analytical approach.

What struck you as the most significant indicators of CAPI?

Did some of them raise specific issue?

Did some of them influence your practice?

Could you tell us of some other potential indicators and why?
Specifically when managing a patient involved applying or not a protocol linked to indicators, what happened?

- In order to comply with the indicator target, have you had to go against what you thought would be best for your patient?

- Specifically can you tell us of a situation where it was difficult for you to find a balance between applying the CAPI protocol with respect to what you perceived/ knew of your patient's views.

If a specific ethical issue was not spontaneously broached during the interviews it was proposed to now use the introduction sentence:

"I will now propose assertions which I invite you to reflect upon":

- Participating in the CAPI scheme inevitably leads to developing strategies such as selecting patients easier to handle in order to increase the probability of success.

- The CAPI scheme pushes for implementing protocols without taking into account the specific situation of the patient, which could lead to bad outcomes.

- Targeting the health domain covered by the CAPI scheme induced disinvesting illnesses not embedded in, but which are essential for the health of your patients.

- Financial incentives for applying CAPI target have a negative impact on the physician/patient relationship since they lead to minimizing patients' views and options with respect to treatment and/or lead to biased information.

- Payment for Performance improves care for patients.

- Paying doctor's for doing what they are supposed to do is derogatory to the medical profession.

- Do you think CAPI may have an impact (and in which direction) on the image and the role of general practice?

Recruitment was difficult. What are the reasons for your colleagues being reluctant to participate in this Focus group?

\section{Interview guide to the CAPI - GPS}

Presentation: This research aims (1) to analyze your perception and knowledge as doctors who did not join the scheme soon after it was launched. (2) To analyze if and how the scheme could have an impact on GPs' practice.

First each participant will be invited to present himself. I suggest you tell us:

1. Your practice location

2. Your practice setting (solo, group practice)

3. The profile of your patients

4. Since when you have been practicing

5. What led you to choose general practice?

6. Other activities linked to doctoring (unions, teaching... In this group none of you joined the CAPI. Why? How did you perceive it? What do you think the objectives of the scheme are? After an overall discussion to see their general perception of the scheme we proceed to a more analytical approach.

What struck you as the most significant indicators of CAPI?

Did some of them raise specific issue?

Do you think some could particularly influence CAPI + GPs practice?

Could you tell us of some other potential indicators and why?

I will propose claims that were raised by doctors who joined the CAPI and I invite you to respond:

- The CAPI scheme augurs a new relationship with the CNAMTS that favors the carrot to the stick.

- The weakness of the sums involved will never lead physicians to adopt practices contrary to what seems to be the most appropriate for the patient.

- It is important to adhere to the tool in order to make it evolve.

- It is an easy way to earn a little more because my practices already correspond to the indicators' targets.

- Indicators contained in the CAPI are really the minimum of quality.

- I can leave when I want to and easily do so.

If a specific ethical issue was not spontaneously broached during the interviews it

was now proposed using the introduction sentence:

"I will now propose assertions which I invite you to reflect upon":

- Participating in the CAPI scheme inevitably leads to developing strategies such as selecting patients easier to handle in order to increase the probability of success.

- CAPI pushes for implementing protocols without taking into account the specific situation of the patient, which could lead to bad outcomes.

- Targeting the health domain covered by the CAPI scheme induced disinvesting illnesses not embedded in, but which are essential for the health of your patients.

- Financial incentives for applying CAPI targets have a negative impact on the physician/patient relationship since they lead to minimizing patients' views and options with respect to treatment and/or lead to biased information.

- Payment for Performance improves care for patients.

- Paying doctors for doing what they are supposed to do is derogatory to the medical profession.

- Do you think CAPI may have an impact (and in which direction) on the image and the role of general practice? 